\title{
Phase separation in the particle-hole asymmetric Hubbard model
}

\author{
Martin Eckstein, ${ }^{1}$ Marcus Kollar, ${ }^{1}$ Michael Potthoff, ${ }^{2}$ and Dieter Vollhardt ${ }^{1}$ \\ ${ }^{1}$ Theoretical Physics III, Center for Electronic Correlations and Magnetism, Institute for Physics, University of Augsburg, \\ 86135 Augsburg, Germany \\ ${ }^{2}$ Institute for Theoretical Physics and Astrophysics, University of Würzburg, Am Hubland, 97074 Würzburg, Germany
}

(Received 31 October 2006; published 2 March 2007)

\begin{abstract}
The paramagnetic phase diagram of the Hubbard model with nearest-neighbor and next-nearest-neighbor (NNN) hopping on the Bethe lattice is computed at half-filling and in the weakly doped regime using the self-energy functional approach for dynamical mean-field theory. NNN hopping breaks the particle-hole symmetry and leads to a strong asymmetry of the electron-doped and hole-doped regimes. Phase separation occurs at and near half-filling, and the critical temperature of the Mott transition is strongly suppressed.
\end{abstract}

DOI: 10.1103/PhysRevB.75.125103

PACS number(s): 71.27. $+\mathrm{a}, 71.30 .+\mathrm{h}$

\section{INTRODUCTION}

One of the major goals of condensed-matter physics during the past decades has been to understand the role of electronic correlations in solids. At the heart of this field lies the metal-insulator transition (MIT) driven by the electronic interaction, the Mott transition. ${ }^{1}$ It occurs in many materials, ${ }^{2}$ in particular, transition-metal compounds such as $\mathrm{V}_{2} \mathrm{O}_{3}$. The underlying physical mechanism for this transition is captured by the single-band Hubbard model, ${ }^{3}$

$$
H=\sum_{i j \sigma} t_{i j} c_{i \sigma}^{\dagger} c_{j \sigma}+U \sum_{i} n_{i \uparrow} n_{i \downarrow}-\mu \sum_{i \sigma} n_{i \sigma} .
$$

Here, $c_{i \sigma}^{\dagger}$ are creation operators for an electron at site $i$ with spin $\sigma, U$ is the local Coulomb repulsion, and $t_{i j}$ are the hopping amplitudes, e.g., $t_{1}$ for hopping between nearest neighbors (NNs) and $t_{2}$ for hopping between next-nearest neighbors (NNNs). In the Hubbard model, the Mott transition can be driven either by an increase of the interaction $U$ (at half-filling), or, for large enough $U$, by a change of the particle density toward one electron per site. The two cases are referred to as bandwidth-controlled Mott transition and filling-controlled Mott transition, respectively. ${ }^{2}$

Much progress in understanding electronic correlation effects in general, and the Mott transition in particular, was made by considering the limit of infinite dimensions. ${ }^{4}$ Dynamical mean-field theory ${ }^{5,6}$ (DMFT) can then be used as a unified framework to study metallic, insulating, and magnetically ordered phases. Both the bandwidth-controlled MIT (Refs. 5 and 7-15) and the filling-controlled MIT (Refs. 5 and 16-21) were studied within DMFT; for $T>0$, the transition is first order. However, in the case of a bipartite lattice and if $t_{i j}$ is restricted to NN hopping, i.e., in the particle-hole symmetric case, the Mott transition at half-filling quite generally does not occur, since it is preempted by the intrinsic weak-coupling instability toward antiferromagnetic (AF) long-range order. To reduce or even avoid this "parasitic" AF phase, one may consider nonbipartite lattices 22,23 or alternatively introduce NNN hopping. The latter introduces a competition which frustrates the antiferromagnetic order. It was proposed that the phase diagram of the single-band Hubbard model with NN and NNN hopping then qualitatively re- sembles that of various correlated-electron materials and thus appears to be the minimal model for such materials. ${ }^{6,24}$

In addition to the frustration of the antiferromagnetic phase, NNN hopping generally makes the Hubbard model particle-hole asymmetric, even at half-filling. This asymmetry, which is a generic property of real materials, ${ }^{25,26}$ will also have an effect on the paramagnetic phase. However, previous DMFT investigations considered a special type of hopping ("random $t_{2}$ hopping") in which the model remains particle-hole symmetric even for $t_{2} \neq 0$, and the paramagnetic phase is not changed at all. $5,9,24,27,28$ By contrast, the effect of nonrandom NNN hopping becomes evident already in the noninteracting system through an asymmetric density of states (DOS), as derived recently by a theoretical technique which is able to treat arbitrary hopping on the Bethe lattice. ${ }^{29,30}$ In the present paper, we proceed to discuss the effects of NNN hopping $t_{2}$ on the phase diagram for finite $U$ and $T$.

As we will show, a striking consequence of NNN hopping is the occurrence of phase separation between metallic and insulating phases even at half-filling. Indeed, in the paramagnetic phase of the Hubbard model, phase separation is known to lead to hysteresis in the density $n(\mu)$ at the Mott transition. ${ }^{19,31}$ For the Hubbard model on a square lattice $(d=2)$, which is closely related to high- $T_{c}$ superconductors, phase separation was found within the dynamical cluster approximation (DCA) for $t_{2}>0$ and $n<1,{ }^{31}$ whereas for $t_{2}=0$, finite-size Monte Carlo calculations ${ }^{32,33}$ provide evidence for a homogeneous state. On the other hand, in infinite spatial dimensions DMFT predicts phase separation already for $t_{2}$ $=0$ and $n \neq 1,{ }^{19,21}$ but not at half-filling. We find that this changes for $t_{1}-t_{2}$ hopping.

For our investigation of the effect of $t_{2}$ hopping on the paramagnetic phase diagram, we use the recently developed self-energy functional approach (SFA) ${ }^{34}$ The SFA is a variational method based on an exact variational principle for the grand potential. ${ }^{35}$ In this scheme, the self-energy of a finite reference system is taken as variational ansatz for the selfenergy of the lattice system. The method is very general and can be applied to finite-dimensional systems ${ }^{36}$ and for various types of interactions. ${ }^{37}$ Within DMFT, the exact result would be recovered for a single-impurity Anderson model (SIAM) with infinitely many bath sites as reference 
systems, ${ }^{38}$ while in practice only a SIAM with a finite number $n_{s}$ of bath sites can be investigated. However, the results converge quickly with increasing $n_{s} \cdot{ }^{39}$ The simplest impurity model considers only a single bath site. Surprisingly, this reference system is already enough to reproduce previous results for $t_{2}=0$ for the Mott transition qualitatively and, sometimes, even quantitatively. ${ }^{38}$ Therefore, we will use this "two-site approximation" (one correlated site and one bath site) also to investigate the case $t_{2} \neq 0$.

The paper is organized as follows. In Sec. II, we give a short description of the SFA and of our method to treat NNN hopping on the Bethe lattice. In Sec. III, NN hopping is studied at and away from half-filling. We calculate the paramagnetic phase diagram as a function of interaction, temperature, and either chemical potential or density. The phase diagrams for $\mathrm{NN}$ and $\mathrm{NNN}$ hopping are discussed in detail in Sec. IV.

\section{MODELS AND METHODS}

\section{A. Self-energy functional approach}

The SFA is based on the variational principle of Luttinger and Ward. ${ }^{35}$ When formulated in terms of the self-energy $\mathbf{\Sigma}$, the basic quantity in this context is the grand-potential functional $\hat{\Omega}[\mathbf{\Sigma}]$, which becomes stationary at the physical self-energy. ${ }^{34}$ It can be written as

$$
\hat{\Omega}[\mathbf{\Sigma}]=\hat{F}[\mathbf{\Sigma}]+\operatorname{Tr} \log \left[-\left(\boldsymbol{G}_{0}^{-1}-\mathbf{\Sigma}\right)^{-1}\right],
$$

where $\hat{F}[\Sigma]$ contains all the information about the interaction, while the noninteracting Green function $\mathbf{G}_{0}$ is considered fixed. (Quantities written in boldface are matrices in single-particle indices and Matsubara frequencies, and $\operatorname{Tr}$ denotes the corresponding trace.) The functional form of $\hat{F}[\mathbf{\Sigma}]$ is generally unknown, apart from the fact that it depends only on the interaction part in the Hamiltonian and not on the kinetic energy. This universality follows directly from various ways in which $\hat{F}[\Sigma]$ or its Legendre transform, the Luttinger-Ward functional, can be constructed. ${ }^{40}$ It allows one to calculate $\hat{\Omega}[\mathbf{\Sigma}]$ exactly for certain variational selfenergies, namely, for all $\boldsymbol{\Sigma}$ that can be considered as the exact self-energy of a reference system with the same interaction. The self-energy, $\boldsymbol{\Sigma}=\boldsymbol{\Sigma}\left(y_{i}\right)$, is varied by varying single-particle parameters $\left\{y_{i}\right\}$ of that reference system. In general, $\boldsymbol{\Sigma}$ can only be calculated for small systems by means of exact diagonalization. Comparing with the selfenergy functional of the reference system and exploiting the universality of $\hat{F}[\Sigma]$, Eq. (2) can be written as

$$
\hat{\Omega}[\boldsymbol{\Sigma}]=\Omega^{\prime}-\operatorname{Tr} \log \left[-\left(\boldsymbol{G}_{0}^{\prime-1}-\boldsymbol{\Sigma}\right)^{-1}\right]+\operatorname{Tr} \log \left[-\left(\boldsymbol{G}_{0}^{-1}-\mathbf{\Sigma}\right)^{-1}\right],
$$

where $\Omega^{\prime}$ and $\boldsymbol{G}_{0}^{\prime}$ correspond to the exact grand potential and the noninteracting Green function of the reference system, respectively. The best approximation for the self-energy is then determined from $\partial \Omega\left[\mathbf{\Sigma}\left(y_{i}\right)\right] / \partial y_{i}=0$.

As noted in the Introduction, the reference system employed in our investigation is a two-site impurity model, ${ }^{38}$

$$
H_{r e f}=U n_{1 \uparrow} n_{1 \downarrow}+\sum_{i=1,2 \sigma} \epsilon_{i} n_{i \sigma}+\sum_{\sigma} V\left(c_{1 \sigma}^{\dagger} c_{2 \sigma}+\text { H.c. }\right) \text {. }
$$

Here, index 1 refers to the correlated impurity and index 2 to the bath site. The on-site energies $\epsilon_{1,2}$ and the hybridization $V$ are taken as variational parameters. As we are interested only in the paramagnetic phase and thus need to consider only spin-independent self-energies, we can take $\epsilon_{i}$ and $V$ to be spin independent as well.

Once a stationary point of $\Omega$ is found, it is tracked in the space of variational parameters $\left\{y_{i}\right\}$ as a function of the external physical parameters (interaction $U$, temperature $T$, and chemical potential $\mu$ ) using local algorithms for the solution of the equations $\partial \Omega / \partial y_{i}=0$. High accuracy is achieved by calculating derivatives of $\partial \Omega / \partial y_{i}$ analytically. ${ }^{39}$ However, close to a first-order transition, it is inconvenient to parametrize the solution by the physical parameters, because in this parametrization several solutions which correspond to various metastable phases coexist. To avoid repeated switching between these solutions, we use an algorithm that treats the physical and variational parameters on equal footing.

\section{B. NN and NNN hopping on the Bethe lattice}

The properties of the lattice enter Eq. (3) only via the term $\operatorname{Tr} \log \left[-\left(\boldsymbol{G}_{0}^{-1}-\boldsymbol{\Sigma}\right)^{-1}\right] \equiv \hat{\Omega}_{\text {latt }}[\boldsymbol{\Sigma}]$. In this section, we show how to evaluate $\hat{\Omega}_{\text {latt }}$ for arbitrary hopping on the Bethe lattice, in particular, for $t_{1}-t_{2}$ hopping. We restrict the analysis to the paramagnetic phase with a spin- and site-independent selfenergy $\Sigma \equiv \Sigma\left(i \omega_{n}\right)$. Generally, the trace in $\hat{\Omega}_{\text {latt }}$ includes a sum over Matsubara frequencies and over all eigenstates $|k\rangle$ of the matrix $\boldsymbol{G}_{0}^{-1}-\boldsymbol{\Sigma}=i \omega_{n}+\mu-\Sigma\left(i \omega_{n}\right)-\boldsymbol{t}$, where $\boldsymbol{t}$ is the matrix of hopping amplitudes. The sum over $|k\rangle$ is then expressed in terms of a sum over lattice sites $i$ and an integral over the local density of states, $\rho_{i}(\epsilon)=\Sigma_{k} \delta\left(\epsilon-\epsilon_{k}\right)|\langle i \mid k\rangle|^{2}$, and the sum over Matsubara frequencies is transformed into an integral over real frequencies, as done in Ref. 38. This leads to the expression

$$
\hat{\Omega}_{\text {latt }}=2 \sum_{i} \int_{-\infty}^{\infty} d \omega f(\omega) \int_{-\infty}^{\infty} d \epsilon \rho_{i}(\epsilon) \Theta[\omega+\mu-\Sigma(\omega)-\epsilon],
$$

where $f(\omega)$ is the Fermi function and the factor 2 accounts for spin degeneracy. For the Bethe lattice with arbitrary coordination number $Z$, there exists a general method to calculate the DOS for any given hopping Hamiltonian. ${ }^{29,30}$ One first introduces hopping Hamiltonians $H_{d}=\Sigma_{d_{i=}=d}|i\rangle\langle j|$ that describe hopping only between $d$ th nearest neighbors. In the limit of infinite connectivity $K=Z-1$, these Hamiltonians must be scaled ${ }^{4}$ according to $\widetilde{H}_{d}=H_{d} / K^{d / 2}$, where $\widetilde{H}_{d}$ retains a nontrivial spectrum for $K \rightarrow \infty$. In particular, the spectrum of the NN hopping Hamiltonian $\widetilde{H}_{1}$ has the well-known semielliptical form $\rho_{0}(\lambda)=\sqrt{4-\lambda^{2}} /(2 \pi)$ in this limit. One may then use a remarkable relation valid for the Bethe lattice, which makes use of its special topological properties. ${ }^{29}$ Namely, every hopping Hamiltonian can be written as a 
function of $\widetilde{H}_{1}$, i.e., for an arbitrary set of hopping amplitudes $\left\{t_{d}^{*}\right\}$ one has

$$
\sum_{d} t_{d}^{*} \widetilde{H}_{d}=\epsilon\left(\widetilde{H}_{1}\right) .
$$

Analytical expressions for $\epsilon(\lambda)$ were derived in Ref. 29. In analogy to crystal lattices, where any translationally invariant Hamiltonian is a function of momentum, $\epsilon(\lambda)$ plays the role of a dispersion relation, and $\lambda \in[-2,2]$ runs over the spectrum of $\widetilde{H}_{1}$. In particular, the dispersion for $t_{1}-t_{2}$ hopping is given by ${ }^{29}$

$$
\epsilon(\lambda)=t_{2}^{*} \lambda^{2}+t_{1}^{*} \lambda-t_{2}^{*} .
$$

The DOS can then be obtained in a straightforward way from the known DOS for $\widetilde{H}_{1}$ by a change of variables. The lattice contribution for arbitrary range hopping then reduces to

$$
\hat{\Omega}_{\text {latt }}=\int_{-\infty}^{\infty} d \omega f(\omega) \int_{-2}^{2} d \lambda \frac{\sqrt{4-\lambda^{2}}}{\pi} \Theta\left[\omega+\mu-\Sigma(\omega)-\epsilon_{t_{d}^{*}}^{*}(\lambda)\right] .
$$

This expression is suitable for numerical evaluation. For $t_{1}$ $-t_{2}$ hopping, the inner integral can be evaluated analytically by solving a quadratic equation. This allows us to obtain $\hat{\Omega}_{\text {latt }}$ with great precision, which is necessary to determine the stationary points of $\hat{\Omega}$ reliably.

We conclude this section with a general remark about the sign of the hopping amplitudes $t_{1}$ and $t_{2}$. For any bipartite lattice, the unitary transformation $c_{i \sigma} \rightarrow(-1)^{i} c_{i \sigma}$, where $(-1)^{i}$ is alternating on the two sublattices, changes the sign of all hopping amplitudes between the two sublattices. Thus, we can assume $t_{1}>0$ without loss of generality. On the other hand, it follows from the particle-hole transformation $c_{i \sigma}$ $\rightarrow(-1)^{i} c_{i \sigma}^{\dagger}$ that $t_{2} \rightarrow-t_{2}$ merely interchanges electron-doped $(n>1)$ and hole-doped $(n<1)$ regimes. Therefore, we can also assume $t_{2}>0$.

\section{NN HOPPING}

The paramagnetic phase diagram of the Hubbard model with only NN hopping $\left(t_{2}=0\right)$ has been studied intensively using DMFT, both for half-filling ${ }^{5,12-14}$ and for finite doping. ${ }^{5,16,17,19-21}$ In this section, we use the two-site approximation to investigate the full parameter space spanned by $U, T$, and $\mu$ and compare with previous results where available.

At $T=0$, the phase diagram has a simple structure (Fig. 1). At half-filling (where $\mu=U / 2$ ), the system is insulating for $U>U_{c 2}$, where $U_{c 2} \approx 5.84 t_{1}^{*}$ for the semielliptic DOS. ${ }^{12}$ For any other filling, the ground state is metallic. In the metal, the charge compressibility $\chi_{\mu}=\partial n / \partial \mu$ remains finite even when the insulator is approached from above or below halffilling, but the chemical potential changes discontinuously from $\mu=\mu_{c 2}^{-}$to $\mu=\mu_{c 2}^{+}$as the filling goes from $n=1^{-}$to $n$ $=1^{+}$. For $\mu_{c 2}^{-}<\mu<\mu_{c 2}^{+}$, the system is insulating and halffilled (Fig. 1). In a region $\mu_{c 1}^{-}<\mu<\mu_{c 2}^{-}$and $\mu_{c 1}^{+}>\mu>\mu_{c 2}^{+}$, both metallic and insulating DMFT solutions exist, with the

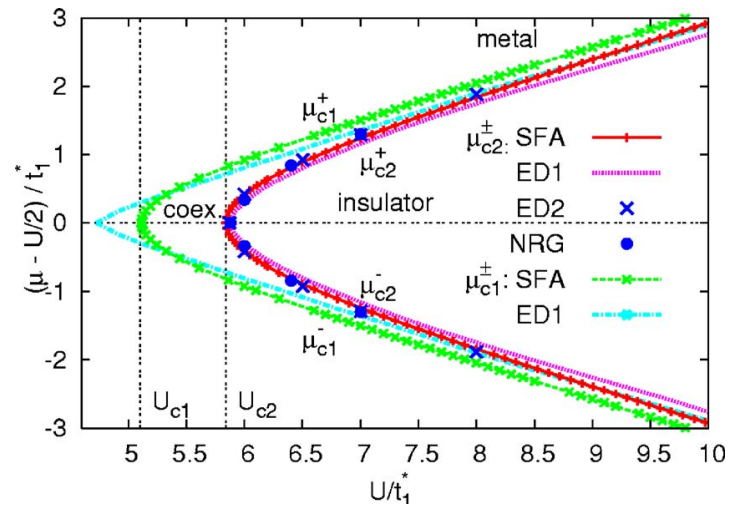

FIG. 1. (Color online) $(U, \mu)$ phase diagram at $T=0$ for NN hopping. Results from two-site SFA (this work) are compared with ED [ED1: eight sites (Ref. 17) and ED2: extrapolation from 11 sites (Ref. 18)] and NRG (Ref. 18). Due to particle-hole symmetry, the phase diagram is symmetric with respect to $\mu=U / 2$.

metallic phase being the thermodynamically stable one. The appearance of the metal at $\mu=\mu_{c 2}^{ \pm}$is related to the development of states inside the Mott gap, whereas the breakdown of the insulator at $\mu=\mu_{c 1}^{ \pm}$occurs when $\mu$ reaches the edge of the gap. ${ }^{16}$ In Fig. 1, we display the phase diagram obtained with the two-site approximation and compare with exact diagonalization (ED). ${ }^{17,18}$ The agreement is remarkably good, in particular concerning the value of $\mu_{c 2}^{ \pm}$. Note that both methods are essentially based on an approximation for the self-energy by a rational function, but the two-site approximation uses a function of much simpler structure, i.e., with fewer poles.

The extension of these results to $T>0$ is best described in the grand canonical ensemble. In Fig. 2, four representative planes in the $(U, T, \mu)$ phase diagram are shown: the $(T, U)$ plane at half-filling ${ }^{38}(\mu=U / 2)$, the $(U, \mu)$ plane at $T=0$ discussed in the last paragraph, and two $(T, \mu)$ planes at fixed interaction. The latter interactions are $U=5.3 t_{1}^{*}$ and $U=6 t_{1}^{*}$, which are slightly smaller and larger than $U_{c 2}$, respectively.

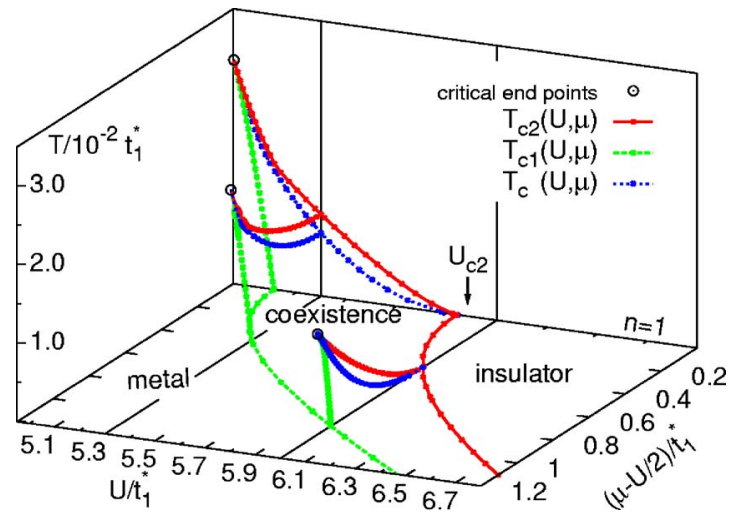

FIG. 2. (Color online) $(U, T, \mu)$ phase diagram for $t_{2}=0$ in twosite SFA. The plot is constructed from the $(U, \mu)$ phase diagram at $T=0$ (Fig. 1$)$, the $(T, U)$ phase diagram at half-filling $(\mu=U / 2)$ as in Ref. 38, and two $(T, \mu)$ sections at fixed interaction $U=5.3 t_{1}^{*}$ and $U=6 t_{1}^{*}$. Due to particle-hole symmetry, the phase diagram is symmetric with respect to $\mu=U / 2$. Only the electron-doped region $(\mu>U / 2)$ is displayed. 


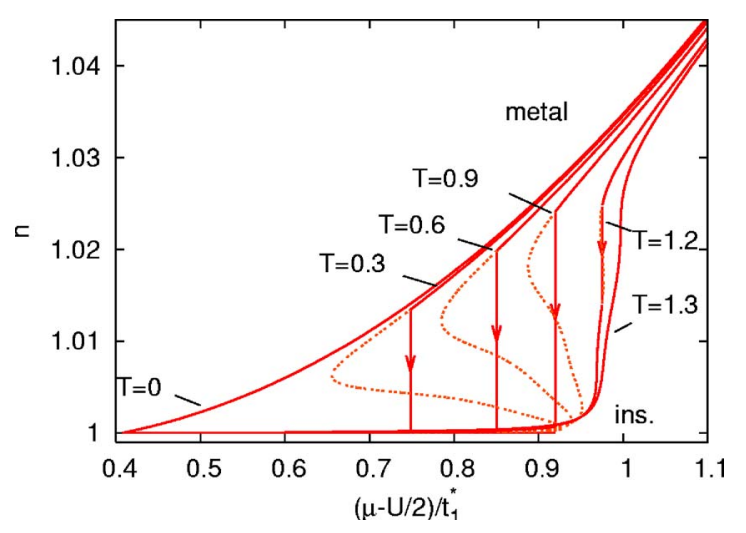

FIG. 3. (Color online) Density as a function of the chemical potential for $U=6 t_{1}^{*}$ and $t_{2}=0$. The temperature values are given in units of $10^{-2} t_{1}^{*}$. The Solid lines correspond to the stable phases, while the dotted lines correspond to the metastable metallic ( $\mu$ $\left.<\mu_{c}\right)$ and insulating $\left(\mu>\mu_{c}\right)$ phases, as well as to a third stationary point of the grand potential functional, which does not correspond to any physical phase because $\chi_{\mu}<0$ (in analogy to the $p-V$ diagram of the van der Waals gas). The discontinuity of $n$ at the transition from the larger value in the metal to a value close to $n=1$ in the insulator is indicated by an arrow.

Our results show that the metallic and insulating regions are separated by a first-order transition surface $T_{c}(U, \mu)$, which coincides with the transition line $T_{c}(U)$ at $\mu=U / 2$ and with $\mu_{c 2}^{ \pm}$at $T=0$. The coexistence region associated with this transition lies between two surfaces $T_{c 1}(U, \mu)$ and $T_{c 2}(U, \mu)$ where the metastable insulating and metallic solutions disappear. They intersect the $T=0$ plane in the lines $\mu_{c 1}^{ \pm}(U)$ and $\mu_{c 2}^{ \pm}(U)$ and meet in a line of second-order critical endpoints.

To detect phase separation, we calculate the density $n(\mu)$ for given $U$ and various $T>0$, as shown in Fig. 3. For the first-order filling-controlled MIT at $T>0$, we find hysteresis; i.e., the densities $n_{c}^{\text {met }}$ and $n_{c}^{\text {iso }}$ in the two phases at the transition $\mu_{c}$ are different. The same behavior has been obtained in quantum Monte Carlo (QMC) DMFT calculations ${ }^{19,21}$ and with the DCA for the two-dimensional Hubbard model..$^{31}$ In the latter work, the similarity of the $n(\mu)$ curves to the $p$ $-V$ isotherms of a van der Waals gas was noted. The insulating solution is characterized by a low charge compressibility and thus corresponds to the (incompressible) liquid, while in the metal $\chi_{\mu}$ stays finite at the transition even for $T \rightarrow 0$.

If the system is prepared at a density within the discontinuities $\left[n_{c}^{i n s}, n_{c}^{\text {met }}\right]$, the free energy is minimized by the formation of a phase mixture with a fraction $x_{\text {met }}=(n$ $\left.-n_{c}^{i n s}\right) /\left(n_{c}^{\text {met }}-n_{c}^{i n s}\right)$ of the metal and $1-x_{\text {met }}$ of the insulator. With QMC, it is difficult to go beyond a calculation of $n(\mu)$ and to determine also $\mu_{c}, n_{c}^{\text {met }}$, and $n_{c}^{\text {ins }}$. Within the SFA, the latter quantities can easily be calculated, since this requires only a comparison of the grand potential (per site) $\Omega$ in the two phases, a quantity that is calculated with high precision anyway. This allows us to go from the $(T, \mu)$, phase diagram to the $(T, n)$ phase diagram, which displays the region between $n_{c}^{\text {met }}(T)$ and $n_{c}^{\text {ins }}(T)$, where the system is unstable against phase separation (Fig. 4). Note that phase separation occurs only away from half-filling. This will be discussed further in the next section.
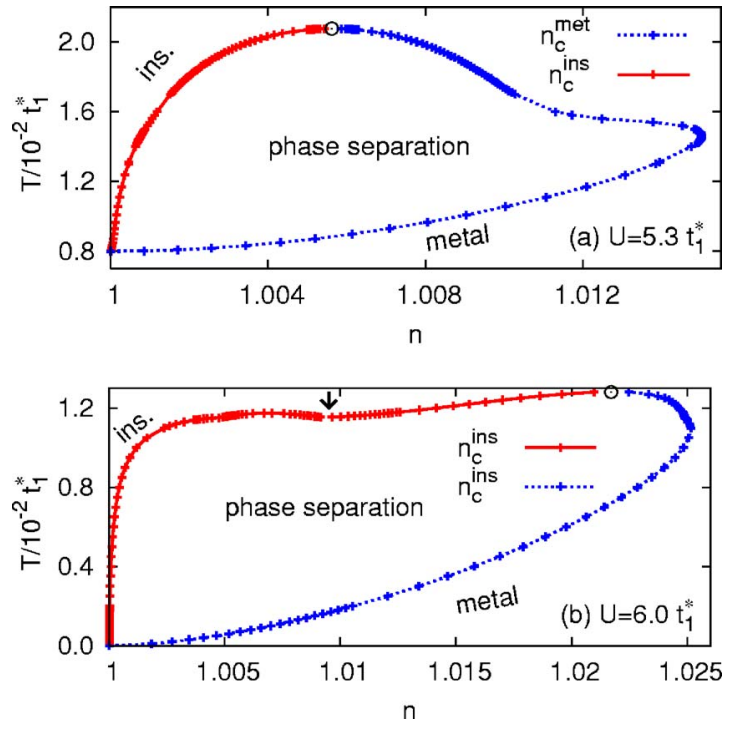

FIG. 4. (Color online) $(T, n)$ phase diagram for $U=5.3 t_{1}^{*}$ and $U=6 t_{1}^{*}$, both for $t_{2}=0$. Small circles indicate the critical point, corresponding to the second-order endpoint of the transition line in the $(T, \mu)$ phase diagram. The small dip in $n_{c}^{\text {ins }}$ for $U=6 t_{1}^{*}$ (arrow) corresponds to an intermediate phase and might be an artifact of the two-site approximation.

\section{NN AND NNN HOPPING}

In the following section, we investigate the influence of NNN hopping on the paramagnetic phase diagram. We focus on the case of $t_{2}^{*} / t_{1}^{*}=3 / 7$. Other values of $t_{2}$ yield qualitatively similar results. The energy scale is set to the square root of the second moment ${ }^{15}$ of the noninteracting DOS, $t^{*}$ $\equiv \sqrt{t_{1}^{* 2}+t_{2}^{* 2}}$.

\section{A. Filling-controlled transition}

For $t_{2} \neq 0$, the surface $T_{c}(U, \mu)$ of the first-order transition in the $(U, T, \mu)$ phase diagram retains the overall shape, as in Fig. 2, but is no longer symmetric with respect to $\mu=U / 2$ due to particle-hole asymmetry. The value of the critical interaction $U_{c 2}$ turns out to be rather insensitive to the value of $t_{2}$ : for $t_{2}^{*} / t_{1}^{*}=3 / 7$, we find $U_{c} \approx 5.45 t^{*}$, in comparison to $U_{c}$ $\approx 5.88 t_{1}^{*}$ for pure NN hopping. Also, the $(T, n)$ phase diagrams look very similar to that of pure NN hopping (Fig. 5). However, particle-hole asymmetry is immediately evident by the very different sizes of the two phase-separated regions, which enclose the Mott transition driven by hole and electron dopings, respectively.

In addition to the phase-separated region, the spinodal curves $T_{c 1}(n)$ and $T_{c 2}(n)$ are included in Fig. 5. To clarify their meaning, let us consider the behavior of the system upon heating at given density $n=n_{0}$, starting from the metallic phase at $T=0$ [arrow in Fig. 5(a)]. When $n_{0}=n_{c}^{m e t}(T)$, the system becomes thermodynamically unstable against the formation of a phase mixture containing the metallic phase $(n$ $\left.>n_{0}\right)$ and the insulating phase $(n \approx 1)$. However, the purely metallic phase $\left(n=n_{0}\right)$ can be superheated up to the spinodal curve $T_{c 2}$. The charge compressibility diverges at the spin- 

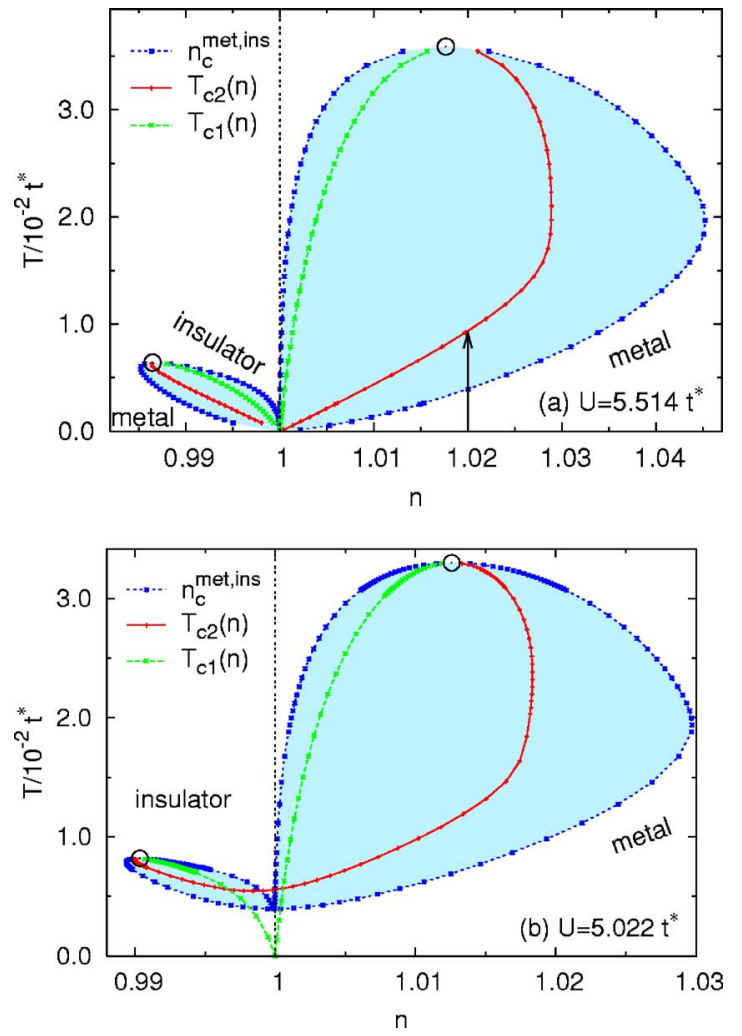

FIG. 5. (Color online) $(T, n)$ phase diagram for $t_{2}^{*} / t_{1}^{*}=3 / 7$. (a) $U=5.514 t^{*}$ and (b) $U=5.022 t^{*}$. The dotted line is the thermodynamic transition line $\left(n_{c}^{m e t}\right.$ and $\left.n_{c}^{i n s}\right)$. Inside this line (shaded region), the system is unstable against phase separation. The arrow in (a) is referred to in the text.

odal curve, indicating increasing density fluctuations in the metastable metallic phase. For $T>T_{c 2}$, a purely metallic solution no longer exists. Similarly, the insulating phase can be supercooled down to $T_{c 1}$.

For the smaller value of the two interactions in Fig. 5, a closer look at the interval around $n=1$ reveals a situation which is different compared to the case of $t_{2}=0$ (cf. Fig. 6): phase separation now occurs at half-filling but disappears at a special density $n_{0} \neq 1$. To understand these numerical results, it is convenient to go back to the $(T, \mu)$ representation.

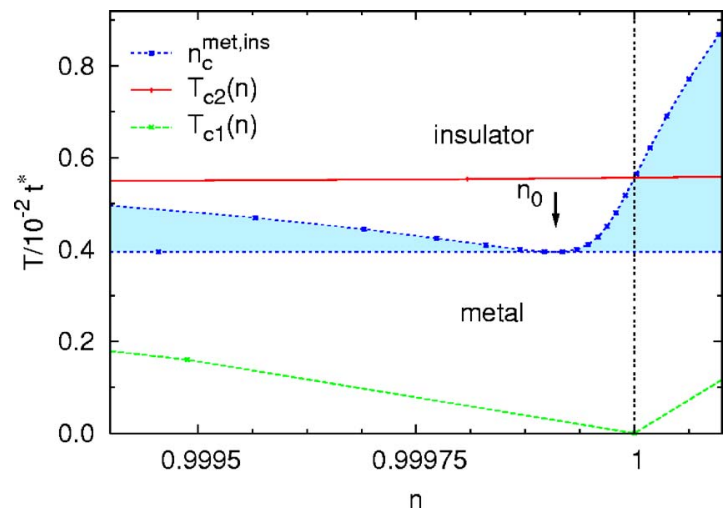

FIG. 6. (Color online) Closeup of Fig. 5(b) near $n=1$ (for a discussion see text).
For given $U$ and $t_{2}=0$, the transition line $T_{c}(\mu)$ has a characteristic U shape (Fig. 2), where the minimum is at $\mu$ $=U / 2$. Precisely at this minimum no phase separation is observed. This is clear from the Clausius-Clapeyron equation,

$$
\frac{d T_{c}}{d \mu}=\frac{n_{c}^{m e t}-n_{c}^{i n s}}{S_{c}^{i n s}-S_{c}^{\text {met }}}
$$

which relates the slope of $T_{c}(\mu)$ to the density $n_{c}^{\text {met }}\left(n_{c}^{\text {ins }}\right)$ and the entropy (per site) $S_{c}^{\text {met }}\left(S_{c}^{\text {ins }}\right)$ in the two phases at the transition. The $\mathrm{U}$ shape has a simple thermodynamic explanation: The stable phase is determined by its lower grand potential (per site) $\Omega=E-n \mu-T S$. For $U<U_{c 2}$, we know that the metal is stable at $T=0$. Now let $\mu=\mu_{1}$ such that $n^{m e t}=1$ for $T=0$. We can expand the difference $\Delta \Omega(\mu)$ $\equiv\left(\Omega^{m e t}-\Omega^{i n s}\right)_{T=0}$ as

$$
\Delta \Omega(\mu)=\text { const }-\frac{\chi_{\mu}^{m e t}}{2}\left(\mu-\mu_{1}\right)^{2}+\cdots,
$$

where we used $\partial \Omega / \partial \mu=-n$ as well as $n_{T=0}^{i n s}=1$. The metal thus becomes more stable as $n$ deviates from $n=1$. However, in DMFT the insulator has a large entropy even at $T=0$, since there is no ordering of the magnetic moments. This entropy gain stabilizes the insulator at a certain temperature $T>0$ and causes the first-order transition. The minimum of $T_{c}$ directly reflects the minimum in $\Delta \Omega(\mu)$. All this remains true for $t_{2} \neq 0$. However, while the minimum of $T_{c}$ lies at $n=1$ as a consequence of particle-hole symmetry for $t_{2}=0$, it shifts to $n_{0} \neq 1$ for $t_{2} \neq 0$.

\section{B. Half-filling $(n=1)$}

Phase separation makes the calculation of the phase diagram for a fixed density considerably more difficult. It is impossible to decide if a system at some given density prefers to assume an inhomogeneous state by investigating only that particular density. Instead, we construct the $(T, U)$ phase diagram for half-filling from several $(T, n)$ phase diagrams (for various $U$ ), as in Fig. 5 .

The first-order line of the particle-hole symmetric case is then found to be replaced by a phase-separated region, as shown in Fig. 7. Within this region, the stable phase is a mixture of two slightly doped phases. Their composition cannot be inferred from Fig. 7 alone and again requires $(T, n)$ sections. A distinctive feature of the phase diagram for pure $\mathrm{NN}$ hopping at half-filling is the triangular-shaped coexistence region (see Fig. 2). By contrast, in the presence of frustration, we find regions of phase coexistence, as well as a small region (close to the critical point) in which both metal and insulator cannot exist as pure phases at $n=1$. This is because the spinodal lines cross once and meet at the critical point, which is indicated by a circle in Fig. 7.

For a closer look at the critical region, consider Fig. 8. When $U$ is lowered, the transition shifts to larger temperatures and becomes more and more asymmetric [Fig. 8(a)] until it finally occurs only in the electron-doped regime [Fig. 8(b)]. For a given $U$, the first-order line $T_{c}(\mu)$ has two critical endpoints. In general the density at these points, i.e., the critical density $n_{\text {crit }}(U)$, is different from 1 . However, when 


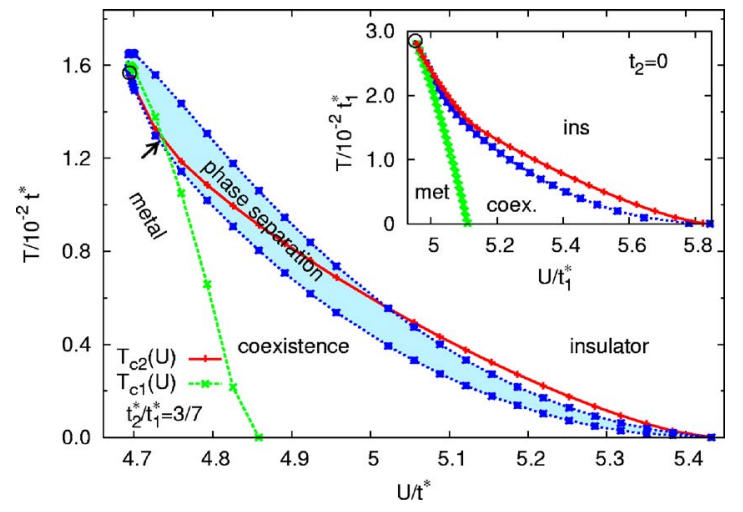

FIG. 7. (Color online) $(T, U)$ phase diagram for half filling and $t_{2}^{*} / t_{1}^{*}=3 / 7$. For comparison, the corresponding plot for pure NN hopping obtained within the two-site approximation is shown in the inset. The circle denotes the critical point. Note that the spinodal curves cross once within the phase-separated region (arrow).

$n_{\text {crit }}=1$ (which occurs between $U=4.69 t^{*}$ and $U=4.7 t^{*}$ in the present case), there is a critical point in the $(T, U)$ phase diagram for half-filling. This point is then characterized by a diverging charge compressibility $\chi_{\mu}$.

Quantitatively, NNN hopping leads to a considerable suppression of the transition region to lower temperatures, while the critical interaction does not change very much. How much this will be modified when one goes beyond the twosite approximation remains to be investigated. Nevertheless, the fact that NNN hopping reduces the Mott transition temperature implies that the competition between paramagnetic and antiferromagnetic phases for $t_{1}-t_{2}$ hopping is more complicated than previously expected.

\section{CONCLUSION}

We employed the self-energy functional approach in combination with dynamical mean-field theory to compute the metal-insulator transition in the $(U, T, n)$ phase diagram of correlated electrons. To this end, the single-band Hubbard model with NN and NNN hoppings on a Bethe lattice was investigated in the paramagnetic phase. For pure NN hopping, we find a transition scenario that is consistent with previous calculations, namely, a first-order filling-controlled metal-insulator transition with phase separation. No phase separation occurs at half-filling, where the transition as a function of the interaction strength is also first order for finite temperatures. NNN hopping strongly modifies this picture and leads to phase separation already at half-filling, i.e., to a discontinuity in $n(\mu)$ such that $n(\mu-\eta)<1<n(\mu+\eta), \eta$ $\rightarrow 0^{+}$. Close to the critical point, the phase diagram for halffilling and $t_{2} \neq 0$ resembles that for $t_{2}=0$ and low doping. We note that for the Hubbard model with pure NN hopping in $d=2$, phase separation was excluded analytically at half-filling, ${ }^{41}$ but this argument does not apply to $d \geqslant 3$.

It is clear that in reality the long-range nature of the Coulomb interaction will counteract the formation of a strongly inhomogeneous charge distribution. Nevertheless, the insta-
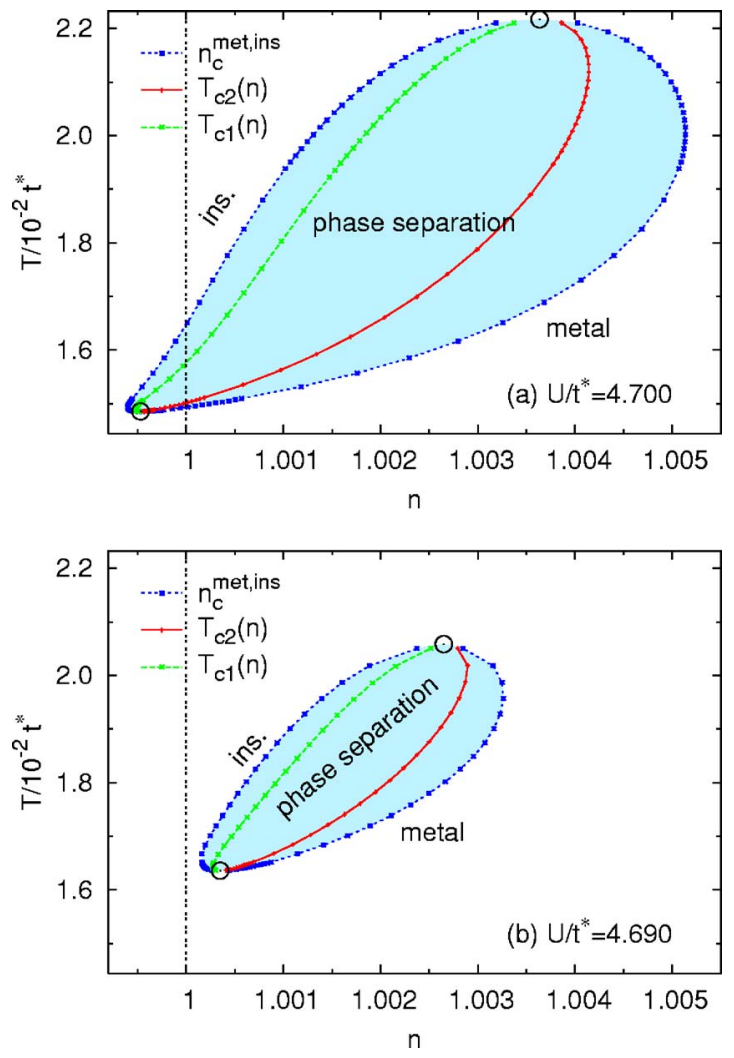

FIG. 8. (Color online) $(T, n)$ phase diagram for $t_{2}^{*} / t_{1}^{*}=3 / 7$. (a) $U=4.7 t^{*}$ and (b) $U=4.69 t^{*}$.

bility with respect to phase separation found in the Hubbard model hints at intriguing physics. Namely, it shows that the inclusion of further interactions will lead to different and interesting phenomena, e.g., more complex ordering phenomena.

Another open question is the influence of NNN hopping on the antiferromagnetic phase. QMC results indicate the suppression of antiferromagnetism. ${ }^{42,43}$ However, to decide whether this will be strong enough to reveal the metalinsulator transition in the paramagnetic phase, the SFA needs to be evaluated for larger reference systems. The present results suggest that frustration not only suppresses the temperature of the antiferromagnetic transition but also that of the Mott transition. Apparently, NNN hopping leads to a more subtle competition between metallic, insulating, and magnetically ordered phases than previously thought. Therefore, it remains a challenging task to understand the physical properties of strongly correlated materials such as $\mathrm{V}_{2} \mathrm{O}_{3}$ in terms of a minimal electronic correlation model.

\section{ACKNOWLEDGMENTS}

We are grateful to Ralf Bulla and Krzysztof Byczuk for discussions. This work was supported in part by Deutsche Forschungsgemeinschaft (DFG) through Sonderforschungsbereich 484 (M.E., M.K., and D.V.) and Forschergruppe 538 (M.P.). 
${ }^{1}$ N. Mott, Metal-Insulator Transitions (Taylor \& Francis, London, 1990).

${ }^{2}$ M. Imada, A. Fujimori, and Y. Tokura, Rev. Mod. Phys. 70, 1039 (1998).

${ }^{3}$ J. Hubbard, Proc. R. Soc. London, Ser. A 276, 238 (1963).

${ }^{4}$ W. Metzner and D. Vollhardt, Phys. Rev. Lett. 62, 324 (1989).

${ }^{5}$ A. Georges, G. Kotliar, W. Krauth, and M. J. Rozenberg, Rev. Mod. Phys. 68, 13 (1996).

${ }^{6}$ G. Kotliar and D. Vollhardt, Phys. Today 57(3), 53 (2004).

${ }^{7}$ M. Jarrell, Phys. Rev. Lett. 69, 168 (1992).

${ }^{8}$ A. Georges and W. Krauth, Phys. Rev. B 48, 7167 (1993).

${ }^{9}$ M. J. Rozenberg, G. Kotliar, and X. Y. Zhang, Phys. Rev. B 49, 10181 (1994).

${ }^{10}$ R. Bulla, Phys. Rev. Lett. 83, 136 (1999).

${ }^{11}$ M. J. Rozenberg, R. Chitra, and G. Kotliar, Phys. Rev. Lett. 83, 3498 (1999).

${ }^{12}$ R. Bulla, T. A. Costi, and D. Vollhardt, Phys. Rev. B 64, 045103 (2001).

${ }^{13}$ N.-H. Tong, S.-Q. Shen, and F.-C. Pu, Phys. Rev. B 64, 235109 (2001).

${ }^{14}$ J. Joo and V. Oudovenko, Phys. Rev. B 64, 193102 (2001).

${ }^{15}$ N. Blümer, Ph.D. thesis, Universität Augsburg, 2002.

${ }^{16}$ D. S. Fisher, G. Kotliar, and G. Moeller, Phys. Rev. B 52, 17112 (1995).

${ }^{17}$ H. Kajueter, G. Kotliar, and G. Moeller, Phys. Rev. B 53, 16214 (1996).

${ }^{18}$ Y. Ono, R. Bulla, A. Hewson, and M. Potthoff, Eur. Phys. J. B 22, 283 (2001).

${ }^{19}$ G. Kotliar, S. Murthy, and M. J. Rozenberg, Phys. Rev. Lett. 89, 046401 (2002).

${ }^{20}$ D. J. García, E. Miranda, K. Hallberg, and M. J. Rozenberg, cond-mat/0608248 (unpublished).

${ }^{21} \mathrm{P}$. Werner and A. J. Millis, cond-mat/0610401 (unpublished).

${ }^{22}$ T. Ohashi, N. Kawakami, and H. Tsunetsugu, Phys. Rev. Lett. 97,
066401 (2006)

${ }^{23}$ K. Aryanpour, W. E. Pickett, and R. T. Scalettar, Phys. Rev. B 74, 085117 (2006).

${ }^{24}$ M. J. Rozenberg, G. Kotliar, H. Kajueter, G. A. Thomas, D. H. Rapkine, J. M. Honig, and P. Metcalf, Phys. Rev. Lett. 75, 105 (1995).

${ }^{25}$ J. E. Hirsch, Phys. Rev. B 65, 184502 (2002).

${ }^{26}$ J. E. Hirsch, Phys. Rev. B 71, 104522 (2005).

${ }^{27}$ R. Chitra and G. Kotliar, Phys. Rev. Lett. 83, 2386 (1999).

${ }^{28}$ R. Zitzler, N. H. Tong, T. Pruschke, and R. Bulla, Phys. Rev. Lett. 93, 016406 (2004).

${ }^{29}$ M. Eckstein, M. Kollar, K. Byczuk, and D. Vollhardt, Phys. Rev. B 71, 235119 (2005).

${ }^{30}$ M. Kollar, M. Eckstein, K. Byczuk, N. Blümer, P. van Dongen, M. H. Radke de Cuba, W. Metzner, D. Tanaskovic, V. Dobrosavljevic, G. Kotliar, and D. Vollhardt, Ann. Phys. 14, 642 (2005).

${ }^{31}$ A. Macridin, M. Jarrell, and T. Maier, Phys. Rev. B 74, 085104 (2006).

${ }^{32}$ A. Moreo, D. Scalapino, and E. Dagotto, Phys. Rev. B 43, 11442 (1991).

${ }^{33}$ F. Becca, M. Capone, and S. Sorella, Phys. Rev. B 62, 12700 (2000).

${ }^{34}$ M. Potthoff, Eur. Phys. J. B 32, 429 (2003).

${ }^{35}$ J. M. Luttinger and J. C. Ward, Phys. Rev. 118, 1417 (1960).

${ }^{36}$ M. Potthoff, M. Aichhorn, and C. Dahnken, Phys. Rev. Lett. 91, 206402 (2003).

${ }^{37}$ N. H. Tong, Phys. Rev. B 72, 115104 (2005).

${ }^{38}$ M. Potthoff, Eur. Phys. J. B 36, 335 (2003).

${ }^{39}$ K. Požgajčić, cond-mat/0407172 (unpublished).

${ }^{40}$ M. Potthoff, Condens. Matter Phys. 9, 557 (2006).

${ }^{41}$ G. Su, Phys. Rev. B 54, R8281 (1996).

${ }^{42}$ M. H. Radke de Cuba, Ph.D. thesis, RWTH Aachen, 2002.

${ }^{43}$ J.-M. Schlipf, Ph.D. thesis, Universität Augsburg, 1998. 